REVISTAS DE LA FAHCE

Faculad de Hunuriads y Cenoirs de la Educadion | tMU

की
Educación Física y Ciencia

ISSN: 1514-0105

ISSN: 2314-2561

revistaefyc@fahce.unlp.edu.ar

Universidad Nacional de La Plata

Argentina

\title{
Prácticas evolutivas de nudos en actividades de escalada en Educación Fisica
}

López, Eduardo Hugo; Reising, María Lilén; Julián, Raúl Horacio; Fernández, Marisa Nélida; Palacio, Mónica Patricia

Prácticas evolutivas de nudos en actividades de escalada en Educación Fisica

Educación Física y Ciencia, vol. 22, núm. 1, 2020

Universidad Nacional de La Plata, Argentina

Disponible en: http://www.redalyc.org/articulo.oa?id=439963095004

DOI: https://doi.org/10.24215/23142561e112

Esta obra está bajo una Licencia Creative Commons Atribución-NoComercial-Compartirlgual 4.0 Internacional. 


\title{
Prácticas evolutivas de nudos en actividades de escalada en Educación Fisica
}

\author{
Assessment Practices of Knot Tying in Climbing Activities in Physical Education \\ Eduardo Hugo López \\ Universidad Nacional del Comahue Bariloche, Argentina \\ eduardohugo.lopez@crub.uncoma.edu.ar \\ DOI: https://doi.org/10.24215/23142561e112 \\ Redalyc: http://www.redalyc.org/articulo.oa? \\ id $=439963095004$
}

Maria Lilén Reising

Universidad Nacional del Comahue Bariloch, Argentina

Raúl Horacio Julián

Universidad Nacional del Comahue Bariloche, Argentina

Marisa Nélida Fernández

Universidad Nacional del Comabue Bariloche, Argentina

Mónica Patricia Palacio

Universidad Nacional del Comahue Bariloche, Argentina

Recepción: 06 Diciembre 2018

Aprobación: 01 Diciembre 2019

\section{ReSUMEN:}

El presente artículo se enmarca en el proyecto de investigación Prácticas pedagógicas de Andinismo en Educación Física. Formación docente y campo profesional, de la Universidad Nacional del Comahue - Centro Regional Universitario Bariloche, Argentina. El propósito es compartir la modalidad de evaluación llevada a cabo con estudiantes de tercer año del profesorado en Educación Física en la cátedra Deportes Regionales Estivales 1. Dicha instancia surge como necesidad de generar un espacio para evaluar los nudos de escalada con el objeto que los/as estudiantes los practiquen, apropien y transfieran a las prácticas de la asignatura logrando mayor autonomía por parte de éstos y seguridad en general durante el desarrollo de las propuestas de escalada. Por otro lado, la información recabada permite pensar estrategias didácticas tendientes a minimizar las dificultades observadas, mejorando así la enseñanza. Se comparten resultados de la evaluación y un análisis cuantitativo de los mismos.

Palabras ClaVE: Evaluación formativa, Andinismo, Educación física, Seguridad.

\section{Abstract:}

This paper is part of the project Pedagogical Climbing Practices in Physical Education. Teacher training and professional field of the National University of Comahue, Bariloche. The purpose of this article is to share the assessment methodology carried out with third-year students of the Physical Education teacher training program, in the subject Regional Summer Sports I. This instance arises through the need to create opportunities to assess the procedure of rock climbing knots to allow students to practise, incorporate and transfer them to their study practice, consequently gaining autonomy in their tying and general safety during the development of the climbing activities suggested. On the other hand, the information gathered will allow the creation of teaching strategies which will tend to minimize the difficulties observed and, therefore, improve teaching methods. Assessment results and its corresponding quantitative analysis are shared.

KEYWORDS: Formative evaluation, Andinism, Physical Education, Safety.

\section{INTRODUCCIÓN}

El trabajo se enmarca en el proyecto de investigación Prácticas pedagógicas de Andinismo en Educación Física. Formación docente y campo profesional ${ }^{1}$, del Centro Regional Universitario Bariloche de la Universidad Nacional del Comahue (Río Negro - Argentina), estudio que analiza y ahonda en las prácticas pedagógicas 
del Andinismo, desde y en el propio campo de la Educación Física tanto en el escenario de la formación docente como en el ámbito profesional; interroga sus discursos, desentraña sus modos particulares de producción, aborda sus lógicas, problemas, propiedades y rasgos distintivos. Existen otros trabajos producidos por el equipo presentados en diversos congresos nacionales ${ }^{2}$. El estudio se posiciona en el campo de la investigación educativa a la que se define desde los aportes de Elena Achilli (2002) como el proceso sistemático de conocimiento de una determinada problemática, que supone un trabajo intelectual de análisis, crítica y confrontación de múltiples informaciones que posibilita construir un objeto de estudio en sus interdependencias y relaciones históricas contextuales. Los referentes teóricos orientan el trabajo en terreno a modo de soporte conceptual con el propósito de generar teoría y construir categorías analíticas que den cuenta de la especificidad de las situaciones analizadas. Desde este posicionamiento no se pretende comprobar teorías sino construir conocimiento para comprender y explicar la problemática planteada.

Las prácticas de Andinismo en el marco del Profesorado en Educación Física, atravesada su enseñanza por dos pilares: la técnica y la seguridad, vienen siendo pensadas, adaptadas y reformuladas para instituirse como contenidos enseñables en el campo de la Educación Física tanto en ámbitos formales como no formales (Goicochea y López, 2016).

Desde esta perspectiva, en el escrito se describe una experiencia pedagógica de evaluación de una práctica de Andinismo, más precisamente de escalada, vinculada con la realización de nudos. Sin embargo dada la importancia que reviste la ejecución correcta de nudos en la actividad, puede aplicarse a diversos escenarios de enseñanza e incluso de práctica de la escalada.

La experiencia descripta se lleva a cabo en la asignatura Deportes Regionales Estivales I del Profesorado en Educación Física de la institución universitaria mencionada. Esta carrera, actualmente de cuatro años de duración, se inicia en el año 1991 con una fuerte impronta en actividades de montañismo lo que le otorga una identidad que se mantiene más allá de las diversas modificaciones del plan de estudio. Desde el año 2004 se proponen dos Trayectos de Formación Específica que definen incumbencias laborales diferenciadas. Uno de ellos denominado Problemática Educativa de la Educación Fisica, se relaciona con actividades de investigación en el área y otro llamado Actividades Regionales de Montaña incorpora en la currícula asignaturas cuyos contenidos están relacionados con el medio natural como prácticas de trekking, escalada, esquí, entre otras. Deportes Regionales Estivales I, es parte de este trayecto y se ubica en el tercer año de la formación. Conforme se explicita en el último plan de estudio, en tanto un modo específico de gestión social del saber (da Silva, 1995), el medio natural se define como eje a modo de categoría que atraviesa al profesorado, desde un abordaje interdisciplinar de la temática, que al mismo tiempo habilita el desempeño de los/as egresados/as en la organización y conducción de prácticas educativas adecuadas a las necesidades del entorno natural.

La experiencia desarrollada en este trabajo, situada en el espacio de la formación universitaria en Educación Física, consiste en la evaluación de ocho nudos de escalada. La pregunta problema que guía la investigación de corte descriptiva con enfoque cuantitativo que se presenta parcialmente en este informe es ¿Cuáles son los errores más frecuentes en la confección de nudos de escalada dentro de la formación docente de profesores de Educación Física en contextos de evaluación?

El objetivo general es describir y cuantificar los errores más frecuentes en la confección de nudos de escalada, en contextos evaluativos durante la formación de docentes de Educación Física. Y los objetivos específicos son: determinar la proporción de aciertos y desaciertos en la confección de nudos de escalada; identificar los errores más frecuentes cometidos en situación de evaluación en la confección de ocho nudos de escalada; determinar el tiempo promedio, máximo y mínimo en la ejecución de nudos de escalada en contextos de evaluación en la formación docente.

A partir del análisis de datos se comparten proporciones de aciertos y desaciertos, dentro de estos últimos se identifican los errores más frecuentes cometidos en cada nudo y se presentan aquellos que menor dificultad evidencian al momento de ser evaluados. Así mismo se releva el tiempo promedio, máximo y mínimo utilizado para cada uno durante la primera instancia. 
Los datos aportados por esta experiencia de evaluación y registro investigativo sirven de base para potenciar las reflexiones pedagógicas de enseñanza, sistemas y modelos de evaluación, así como también propician modificaciones concretas en las prácticas de formación docente, para propender a la mejora de la calidad educativa.

Si bien la experiencia relatada está situada en el profesorado, la detección de errores frecuentes en la ejecución de nudos de escalada podría transferirse a la enseñanza en otros escenarios. Dada la novedad de las actividades de escalada vinculadas con la Educación Física presente desde la década de 1990 en la formación profesional de docentes en Argentina, España y varios países europeos v.g. Austria, Alemania (Palacio, 2019 , pp.52-55) y la reciente incorporación de la Escalada como deporte olímpico, consideramos que el trabajo pretende realizar un aporte al campo disciplinar de la Educación Física en contexto amplio.

\section{LAS PRÁCTICAS EVALUATIVAS}

Desde la asignatura Deportes Regionales Estivales I se entiende a la evaluación como práctica compleja atravesada por múltiples variables tales como el objeto, los sujetos, momentos e instrumentos de evaluación, el contexto institucional y sociohistórico entre otros. De igual manera es definida como dimensión constitutiva e inherente a la enseñanza, es decir, se aleja de la idea de evaluación en tanto apéndice o instancia final del proceso formativo.

En este enfoque, la evaluación se identifica como práctica política y ética que genera alto impacto en las trayectorias estudiantiles. Propicia el diálogo y la comprensión tanto de los aprendizajes como de las prácticas docentes. Si bien es un proceso que va sucediendo, resulta relevante identificar momentos clave o específicos destinados a las prácticas evaluativas a modo de evidencia de logros que dan lugar a la toma de decisiones en pro de la mejora de los aprendizajes.

Se proponen diversos formatos de evaluación, así como variadas instancias de auto, co y heteroevaluación que requieren la implicancia y la participación comprometida de los/as estudiantes.

Se plantea una evaluación desde la perspectiva de la retroalimentación centrada en las interacciones dialogadas formativas (Anijovich, 2010) y como oportunidad para el aprendizaje. Implica reconocer la distancia entre el punto en el que se encuentra y aquel donde es necesario llegar, pudiendo identificar las estrategias necesarias en ese trayecto. Este tipo de evaluación formativa requiere definir con claridad criterios, indicadores e instrumentos pertinentes a la finalidad de la práctica evaluativa.

En esta experiencia en particular, la práctica evaluativa de los nudos de escalada se articula con una instancia de acreditación, es decir, con el reconocimiento institucional de los aprendizajes constatado a través de un instrumento particular y comunicado por medio de una escala conceptual convencional (Steiman, 2008). Es una evaluación individual, presencial que requiere implementar una ejecución. Si bien se evalúa un procedimiento, no se espera una única forma de resolución, sino que se promueven formas de resolver personales. Entre las posibles ejecuciones se contemplan espacios de devolución y andamiaje docente y frente a los procedimientos de resolución inadecuados se trabaja desde la metacognición sobre los modos adoptados y las decisiones tomadas. La detección de los inconvenientes y errores en la realización de los nudos otorga insumos para mejorar las intervenciones docentes y la propuesta de enseñanza de estos.

\section{LOS NUDOS EN LAS PRÁCTICAS DE ESCALADA}

La utilización de nudos en las actividades de escalada se torna un componente fundamental para llevar a cabo la práctica. El poder elegir las opciones más convenientes para cada situación a partir de las prestaciones, los pro y contra de cada nudo, así como su correcta realización, forman parte constitutiva de la actividad, no sólo 
a nivel de la seguridad sino también para favorecer el desarrollo autónomo de la escalada por parte de cada estudiante/escalador.

Los nudos permiten usar cuerdas, cordines y cintas para una diversidad de propósitos. Escaladores, montañistas y profesores en Educación Física suelen recurrir a diferentes opciones de nudos a fin de realizar apropiadamente la práctica con la mayor seguridad posible. Si bien algunos nudos comparten funciones, la elección de este no tiene que ver con una cuestión de gusto personal, sino que está relacionada con aspectos técnicos tales como la cantidad de cuerda/cinta/cordín a utilizar, la manera de ajustarse ante la carga y la resistencia a la misma, entre algunos.

El abordaje para la enseñanza de nudos de escalada en el Profesorado en Educación Física se realiza a partir de una clasificación según sus usos y funciones, distinguiéndose cinco grupos (Schubert y Stockl, 2007; Raleigh, 1999; Núñez, 2005). Si bien en varios de los grupos pueden incluirse otros nudos, aquí se mencionan exclusivamente los enseñados en la asignatura que fueron motivo del presente artículo tal como se muestran en la figura 1.

Nudos de fijación: son aquellos que se utilizan para vincular el arnés (escalador/a) a la cuerda o para fijar o anclar la cuerda (sea en el extremo o no) a un anclaje artificial o natural. Los nudos que se incluyen en este grupo son el 8 doble, 8 reconstruido, estático/ballestrinque, junto a otros.

Nudos de unión: posibilitan unir cuerdas de igual o similar diámetro, realizar anillos con cordines y cintas. Dentro de este grupo se incluye para uso de cuerdas y cordines el doble pescador, 8 reconstruido y para el caso de la cinta el nudo de cinta plana junto a otros.

Nudos de encordamiento: se utilizan para vincular el/la o los/as escaladores/as a la cuerda mediante el uso del arnés. A este grupo corresponde el 8 reconstruido y 8 doble junto a otros.

Nudos autoblocantes: funcionan como un reaseguro para el/la escalador/a utilizado preferentemente durante el desplazamiento por cuerdas fijas y/o rappel. Dentro de este grupo, en la materia se enseña el prusik.

Nudos especiales: por su uso y función no son compatibles con ninguna de las mencionadas clasificaciones. Aquí se encuentran el bulín especial (utilizado para vincular la cinta de autoseguro del/de la escalador/a al arnés) y el nudo dinámico (para dar seguro al/a la escalador/a cuya posibilidad de frenado se produce por rozamiento). 


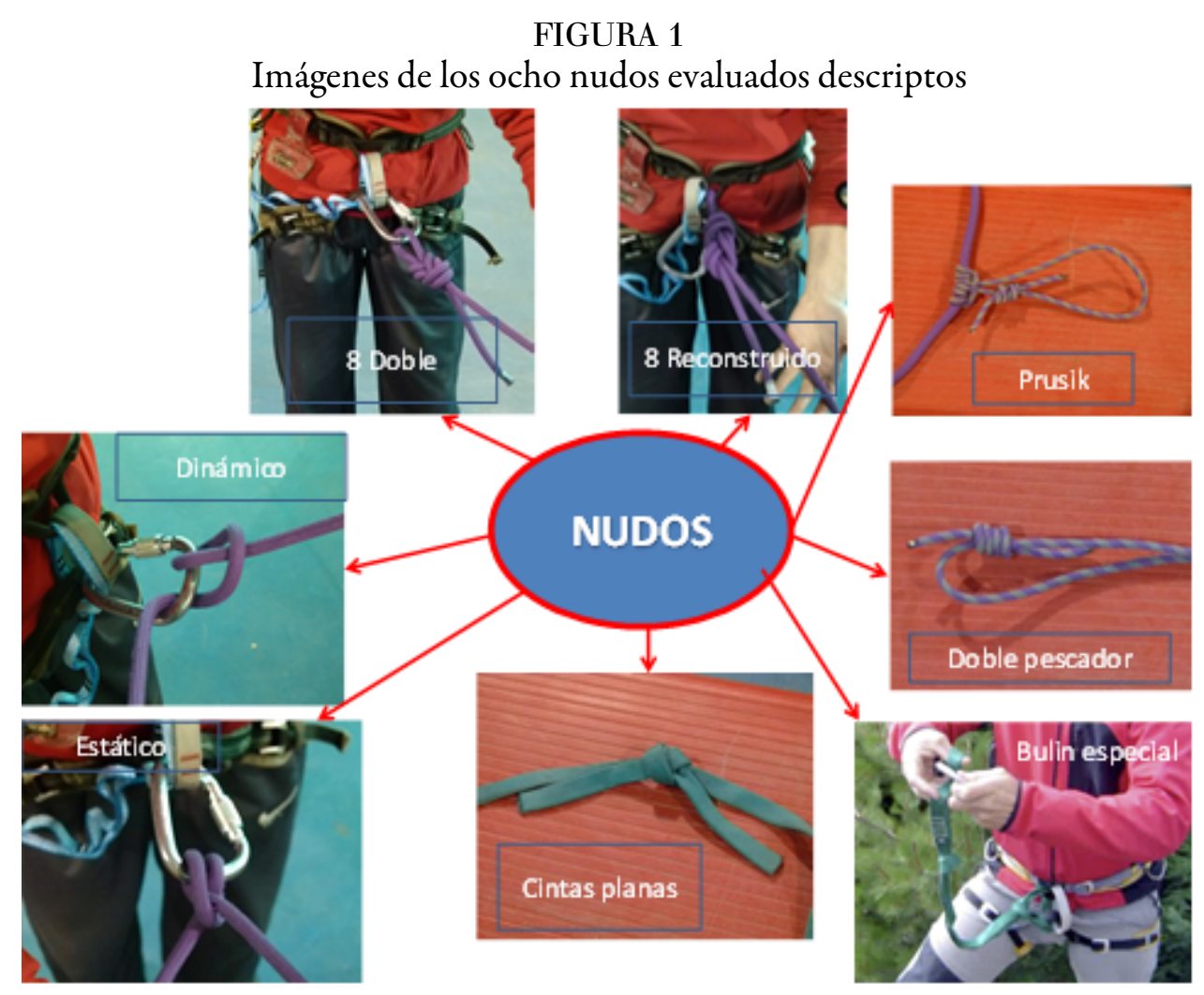

\section{Metodología}

\section{La propuesta de evaluación de nudos de escalada}

Si bien el proyecto de investigación que encuadra este trabajo complementa enfoques cuali y cuantitativo, a modo de vía de profundización que abre a nuevos planteamientos teóricos y potencian el trabajo, en esta oportunidad se efectúa un primer recorte desde una perspectiva cuantitativa. Se selecciona una muestra no probabilística, intencional (Ander Egg, 1993) integrada por 28 estudiantes cursantes de la asignatura Deportes Regionales Estivales I pertenecientes a la cohorte 2017. Durante los meses de agosto, septiembre y octubre de dicho año, se realiza una observación cuantitativa, entendiéndola como el registro válido y confiable de conductas manifiestas (Hernández Sampieri, Fernández Collado y Baptista Lucio, 2014). Mediante la utilización de una grilla de examen, se registran datos acerca de las evaluaciones que cada estudiante realiza en cada uno de los nudos. En la búsqueda de evidencias de los aprendizajes, entendiéndolas como certezas claras y manifiestas, que permiten la demostración de algo (Anijovich y Cappelletti, 2017) se diseñan los sistemas con los que se realiza la evaluación de los saberes técnico-prácticos de la escalada.

Para la ejecución y aprobación de estos, no se establece un límite en la cantidad de instancias de evaluación, sólo se determina una fecha tope al cierre de la cursada. La finalidad de la evaluación es que cada estudiante comprenda y logre realizar correctamente la totalidad de los nudos requeridos durante la misma. Dicho requerimiento se fundamenta a partir del eje de seguridad ya que la prolijidad y la buena confección de los nudos aumentan las condiciones de seguridad no sólo individual sino fundamentalmente del grupo. Dicho de otro modo, la correcta ejecución permite cuidarse mutuamente durante las prácticas de Andinismo y resulta más fácil controlar los nudos que tienen una estructura correcta. En la enseñanza de los nudos se propicia la práctica de estos a través del armado y desarmado, así como la sistemática revisión de las estrategias de ejecución y de los resultados de los nudos realizados. 
Los criterios de la evaluación que constituyen las categorías observadas son pertinencia y finalización correcta, los indicadores propuestos son largo del chicote, tamaño del seno, estructura encastrada, cinta rizada entre otros.

Las variables relevadas son: Datos contextuales, Nombre y apellido, Sexo, edad, Número de intento, ejecución (correcta/ incorrecta) tipo de error y tiempo de ejecución.

La modalidad de la evaluación consiste en realizar los ocho nudos con un tiempo máximo de cinco minutos. Este tiempo se estipula al triplicar el estimado utilizado por expertos en la práctica. Se explicita que los cinco minutos son contabilizados solamente en aquellos momentos en los que el/la estudiante se encuentra desarrollando cada uno de los nudos. Entre cada ejecución se detiene el tiempo y se habilita un espacio de fecundo intercambio entre docentes y estudiantes, generando un escenario de aprendizaje a partir de la evaluación realizada. En aquellas situaciones en las que se realiza de manera incorrecta algún nudo, se requiere efectuarlo en una próxima instancia de evaluación. Para esa situación se considera un tiempo parcial, definido previamente, promediando los tiempos utilizados por los/as estudiantes que aprobaron cada nudo. Ante errores de procedimiento se reitera la evaluación tantas veces como sea necesario a fin de garantizar su correcta resolución.

La evaluación de nudos se implementa en la cátedra desde hace cuatro años. Responde a reflexiones generadas a partir de la identificación de reiteradas dificultades en las prácticas de realización de estos. La propuesta ha sufrido diversas modificaciones en función de revisiones efectuadas en las intervenciones docentes.

Algunas preguntas orientadoras del relevamiento de las evaluaciones son:

¿Qué proporción de estudiantes rinden correctamente cada nudo en la primera instancia de evaluación? ¿Qué proporción de estudiantes requieren más de una instancia para realizarlo correctamente?

¿Cuáles son los errores más frecuentes observados en la realización de cada nudo?

¿Cuál o cuáles son los nudos que menor y mayor nivel de dificultad presentan al ser evaluados?

¿Qué tiempo promedio requiere la ejecución de cada nudo?

¿Cuál es el tiempo máximo y mínimo que se utiliza para cada nudo en la primera instancia?

La evaluación de nudos es rigurosa, se consideran aprobados únicamente aquellos que cumplen en su totalidad con la correcta terminación.

\section{Resultados y Discusión}

A partir del análisis de las evaluaciones implementadas con los/as estudiantes de la muestra es posible afirmar que:

Las proporciones de acierto, de mayor a menor para cada nudo son: el bulín especial, el total de los/las estudiantes lo realiza de manera correcta en la primera instancia; el prusik, el nudo dinámico y el nudo estático se efectúa correctamente en la casi totalidad de los casos en la primera instancia ( 26 de 28 estudiantes). El doble pescador es realizado correctamente por 25 de 28 estudiantes en la primera instancia; el 8 doble obtuvo un acierto de dos tercios en la primera instancia. El nudo de cintas planas presenta un acierto de más de la mitad de los estudiantes evaluados. El nudo que menos efectividad conlleva es el 8 reconstruido, el éxito sólo alcanza 17 de 28 estudiantes en la primera instancia.

Errores detectados en la confección de los nudos:

A continuación, (figura 2) se presentan los errores más frecuentes ordenados de acuerdo a la dificultad que presentan: 
FIGURA 2

Instancia de evaluación 1 . Nudo 8 reconstruido

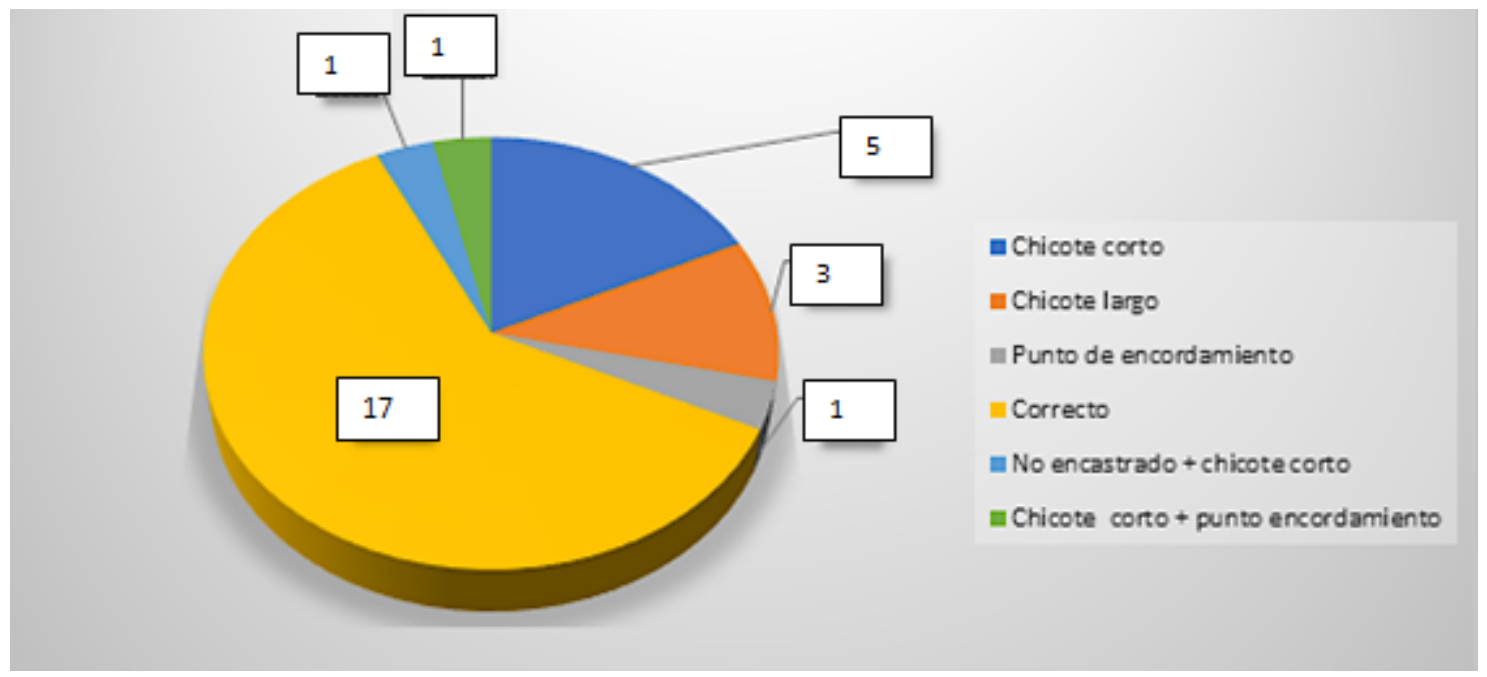

Fuente: Elaboración propia

Los números indican cantidad de estudiantes. El error más frecuente es el de chicote corto que implica el eventual riesgo de que se desarme el nudo. En el caso del chicote largo, error que le sigue en frecuencia, conlleva a la incomodidad del chicote suelto y a la posible confección de un nudo extra conocido como remate que puede ocasionar confusiones de no visualizar bien el nudo. Le siguen en orden de importancia el punto de encordamiento, el nudo no encastrado; en el primero es fundamental conocer cada arnés y su punto central de encordamiento y en el segundo caso al no estar encastrado pierde un $10 \%$ de resistencia en relación al material no anudado.

A partir de las dificultades observadas, se promueven instancias de revisión que constituyen dispositivos de enseñanza compartidos entre docentes y estudiantes y que apuntan a la metacognición mencionada en el inicio del presente artículo. Se analizan y buscan estrategias para definir correctamente la cantidad de cuerda a utilizar en la confección del nudo, se considera el recorrido de cuerda al iniciar la reconstrucción del mismo y se promueve que el/la estudiante al momento de observar que el nudo quedo incorrecto pueda hacer lectura de los errores y corregirlo sin necesidad de desarmarlo.

FIGURA 3

Instancia de evaluación 1 . Nudo cinta plana

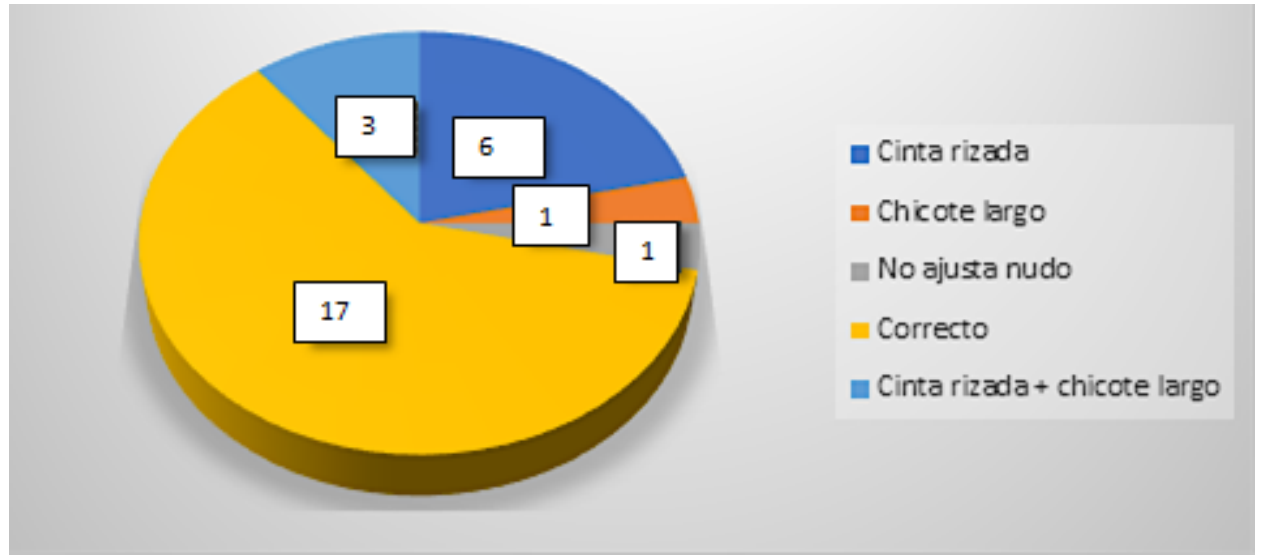

Fuente: Elaboración propia 
El error más frecuente (figura 3). Los números indican cantidad de estudiantes) es la cinta rizada, implica una modificación del material que impacta sobre la resistencia. El error de chicote largo no va en detrimento de la seguridad, pero genera incomodidad en su uso. El tercer error en orden de frecuencia es el no ajuste del nudo que implica riesgos en la seguridad de carácter más serio. Es importante ajustar los cuatro cabos individualmente y no de a par, evitando así el desarme del nudo.

A partir de las dificultades observadas se proponen instancias de revisión. En relación al error de cinta rizada se analiza el orden de las acciones en la confección partiendo desde un nudo de cinta simple, luego recorrer la totalidad de la cinta para verificar que no queden rizos y posteriormente reconstruir el nudo. De igual manera se examina la cantidad de cinta utilizada para que los chicotes queden del largo correcto.

\section{FIGURA 4}

Instancia de evaluación 1 . Nudo 8 doble

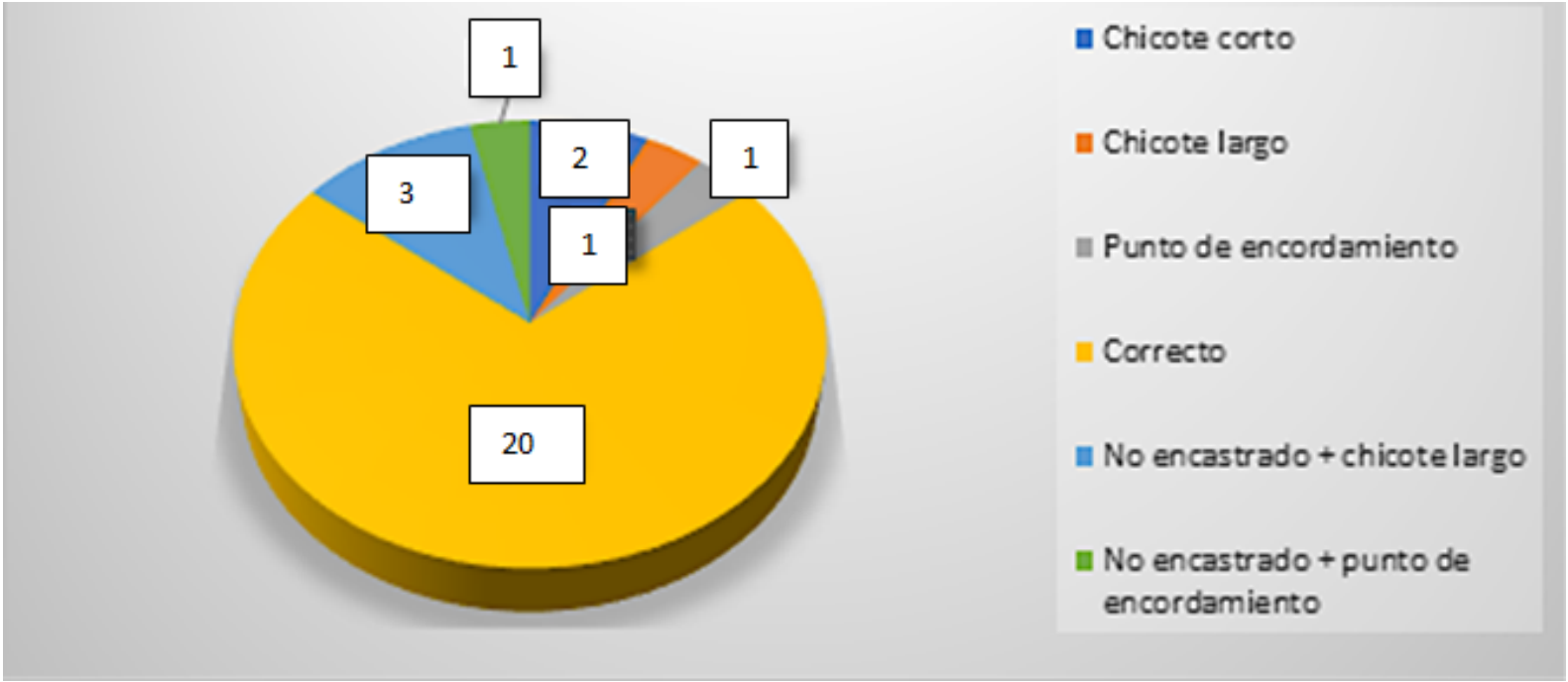

Fuente: Elaboración propia.

Los errores de no encastrado + chicote largo y chicote corto son los más frecuentes (Figura 4) y responden a las mismas consideraciones que se efectuaran al nudo 8 reconstruído. Le siguen en orden de importancia los errores de no encastrado + punto de encordamiento combinados. Los números del gráfico indican cantidad de estudiantes.

En relación a las dificultades observadas se proponen revisiones. En cuanto al error no encastrado se acompaña al/la estudiante para que pueda identificar cuál de los tramos de cuerda se deben revisar y acomodar. Se examina la cantidad de cuerda utilizada para que el chicote y el seno queden con el tamaño correcto. 
FIGURA 5

Instancia de evaluación 1 . Nudo doble pescador

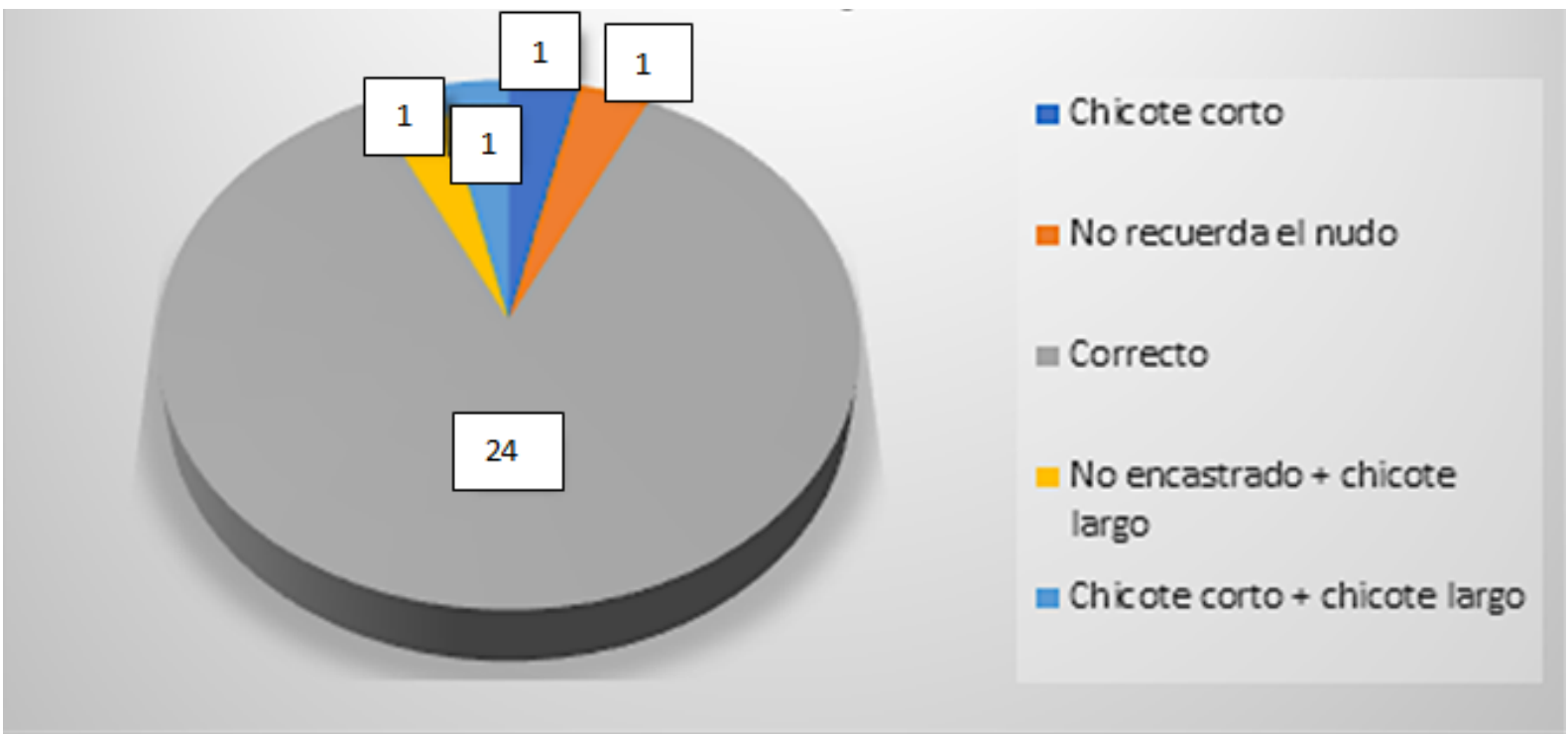

Fuente: Elaboración propia.

La particularidad de este nudo (Figura 5) es que posee dos terminaciones en las que se puede suceder que de un lado sobre chicote y del otro falte. Un caso no recuerda el nudo y en otro aparece el chicote corto en ambos extremos. Éste es el error más riesgoso ya que puede ocurrir que el nudo se desarme. Los números indican cantidad de estudiantes.

Respecto a los errores que se dan en los otros nudos de mucha menor frecuencia ocurre que en los nudos dinámico y estático se los confunde entre sí y en un caso se confunde el estático con el nudo de alondra. Durante la práctica, si se realiza el nudo estático en lugar del dinámico la acción se vuelve inviable. No se trata de un error grave en términos de seguridad pero no se puede realizar la actividad. Si el caso fuese inverso, se hace un nudo dinámico en vez de un nudo estático, sí resulta grave en términos de seguridad ya que si se necesita hacer un estático es porque se quiere fijar la cuerda y/o cordín. El dinámico permite el desplazamiento de la cuerda o cordín.

La proporción de errores en el nudo prusik es muy baja, en general se deja el nudo de unión en el extremo donde luego se unirá el cordín al mosquetón de seguro, por ejemplo y otro caso es no dejar encastrado el nudo. Esto es importante porque el nudo podría bloquear menos al no estar perfectamente ejecutado. En el bulín especial no se identifican errores en la primera instancia de evaluación.

Durante la primera evaluación se incorpora la variable tiempo, lo que genera la toma de conciencia de la necesidad de mayor práctica. En la ejercitación del nudo se propicia la reflexión por parte de los/las estudiantes a fin de pensar y explorar estrategias personales para su resolución.

A continuación, se presentan los tiempos promedio de resolución de cada nudo: 
FIGURA 6

Instancia de evaluación 1. Tiempo de resolución en segundos

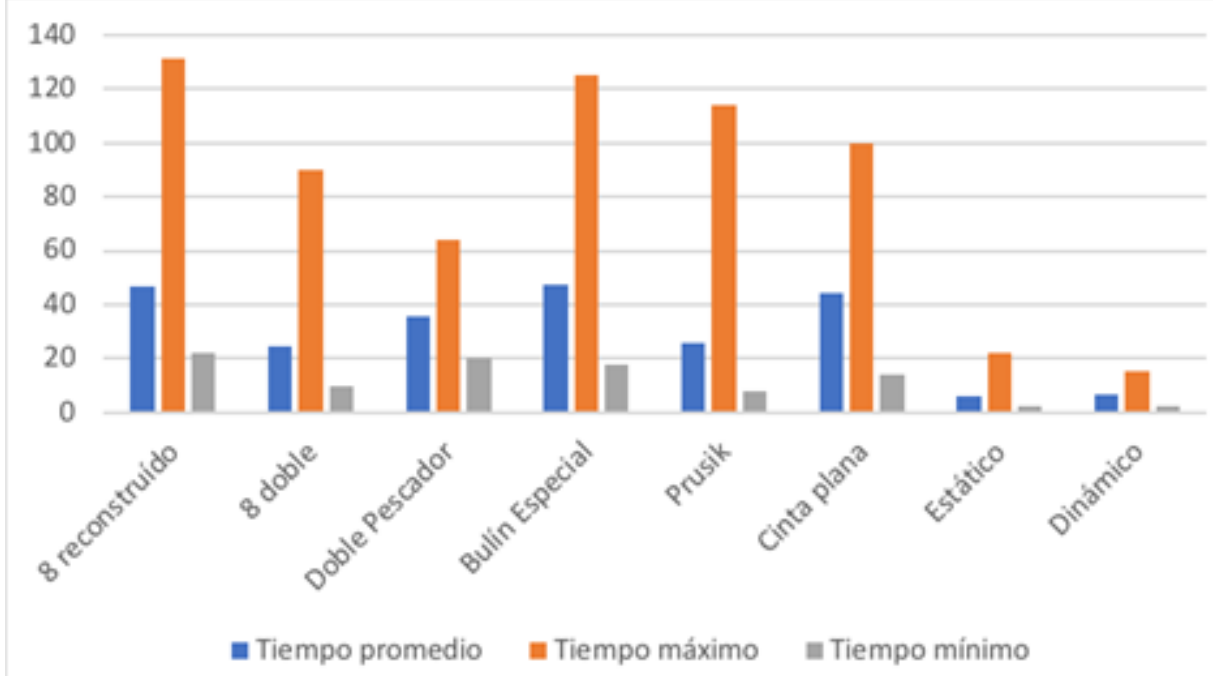

Fuente: Elaboración propia.

Los nudos de mayor velocidad de resolución (Figura 6) son el estático y el dinámico. Los que presentan mayor tiempo de resolución son el 8 reconstruido, el bulín especial y el de cinta plana. Se destaca que si bien poseen tiempos de resolución similar el bulín especial no presenta errores en su ejecución. Los casos de 8 doble y prusik denotan similar tiempo de resolución promedio, sin embargo, en el caso del prusik el tiempo máximo utilizado es superior al anterior. Otro dato significativo resulta que el nudo que requiere un tiempo máximo de ejecución mayor es el 8 reconstruido.

\section{Conclusiones}

Cada nudo posee una única manera de terminación que optimiza su resistencia, no obstante, durante el armado se observan distintas formas que tanto estudiantes como profesores adoptan según su habilidad y facilidad para comprender su confección. En el nudo, tanto cintas, como cordines y cuerdas deben soportar además de las fuerzas puramente traccionales, fuerzas de plegado, de presión y de cizallamiento, las cuales debilitan la resistencia de todas las fibras textiles (Schubert y Stockl, 2007).

La importancia en su correcta terminación se basa fundamentalmente en dicho aspecto ya que se estima, de manera práctica y en general, que la resistencia a la rotura de un cordín o una cinta con nudo es igual a la mitad de la resistencia sin nudo (Schubert y Stockl, 2007), entendiendo que dicho nudo esté realizado de una manera correcta. Por otro lado, el habituarse a concluir cada nudo atendiendo a todos los detalles en su terminación facilita el poder identificar en otro/a un posible error en su confección que pone en riesgo la integridad física propia y/o del grupo. En este sentido, aprender y ejecutar los nudos correctamente (Figura 7 y 8), facilita durante el chequeo que se realiza entre escalador/a y asegurador/a o entre los/as involucrados/ as en la práctica, visualizar y detectar posibles errores. 


\section{FIGURA 7}

Práctica de escalada en roca (ejecución de nudos) Deportes Regionales Estivales 1 en la zona de Laguna Negra, Parque Nacional Nahuel Huapi, Patagonia, Argentina

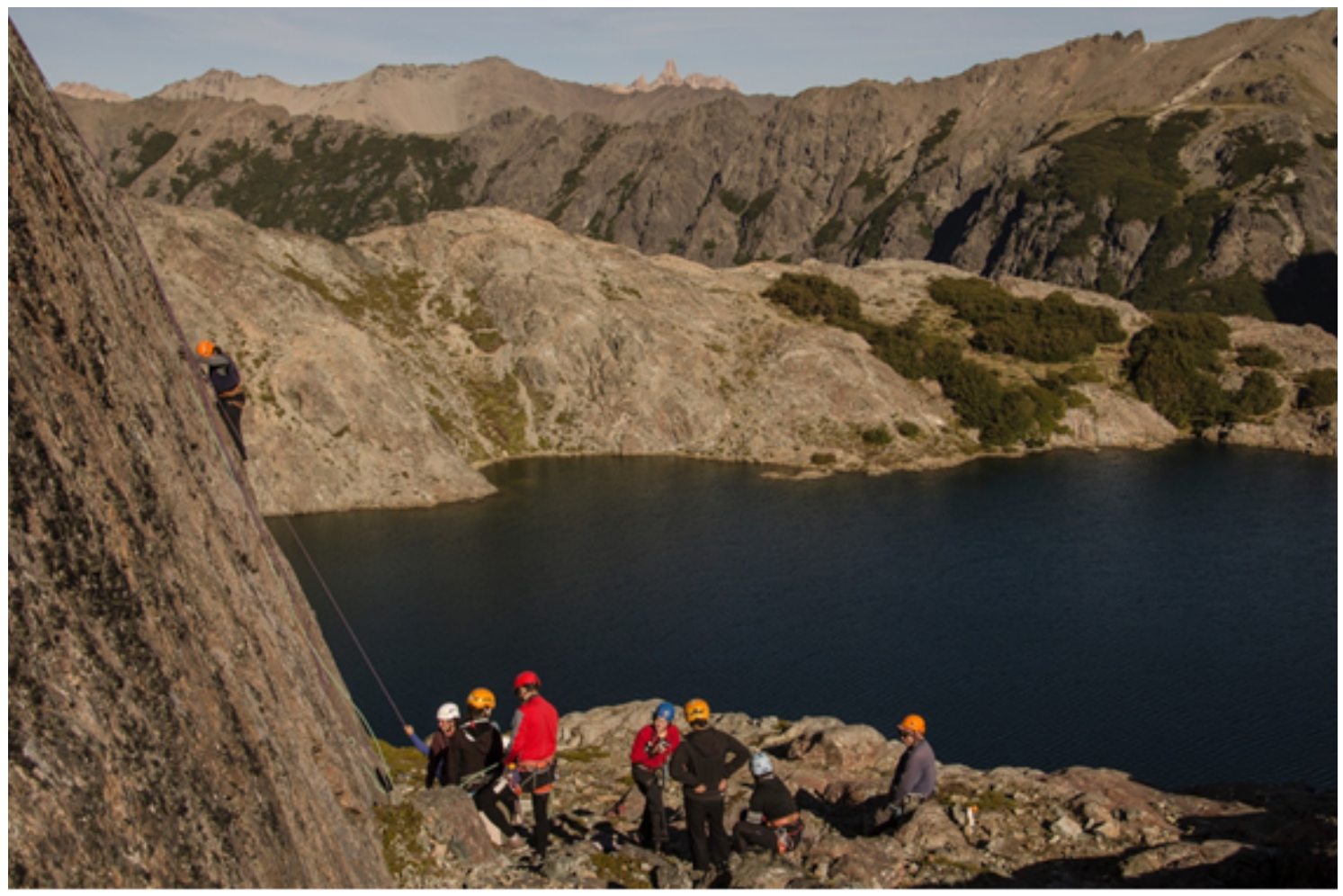

Foto: Eduardo Lopez.

FIGURA 8

Ascensión al Volcán Osorno, Chile, Deportes Regionales Estivales 2

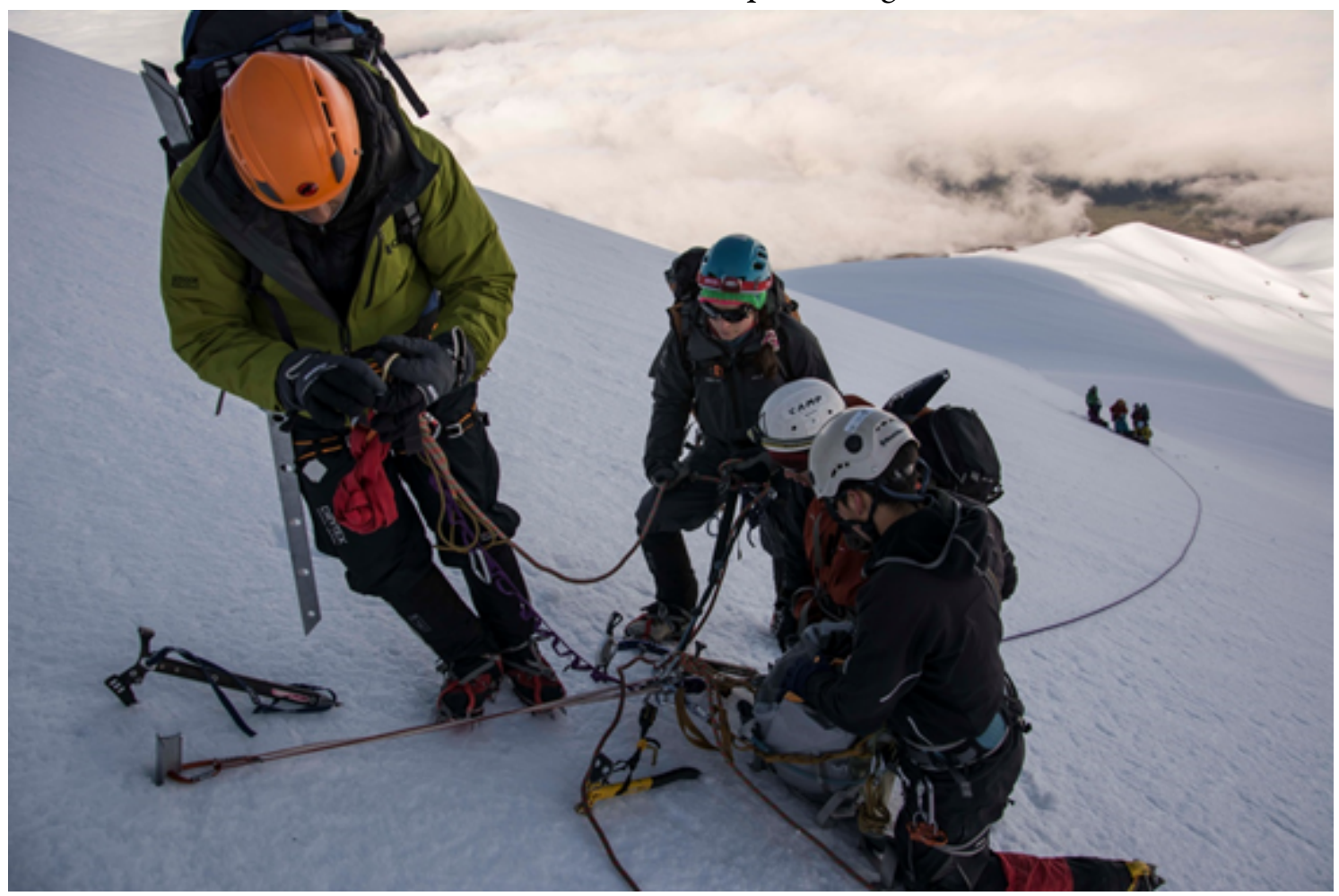


Foto: Eduardo Lopez.

La redundancia se manifiesta como principio de enseñanza de los nudos no como repetición rutinaria y mecánica sino en términos de tratamiento espiralado que otorga continuidad, progresión y profundización de la práctica (Ron y Fernández, 2017).

La detección de los inconvenientes y errores en la resolución de los nudos proporciona insumos para mejorar la enseñanza de estos. Conforme se refleja en los datos presentados, los nudos que presentan mayor proporción de error ( 8 reconstruido y cinta plana), evidencian la necesidad de atender a reformular estrategias didácticas.

De las evaluaciones realizadas se desprende que aquellos/as estudiantes que rinden en un momento elegido a voluntad presentan menor proporción de errores y mayor seguridad en la ejecución que quienes lo realizan hacia el momento establecido como límite de esta.

La incorporación de la variable tiempo en la evaluación motiva la práctica de los nudos y propicia la identificación de estrategias personales para su resolución. No obstante, durante las prácticas de escalada no es una variable determinante, ya que se prioriza la dimensión seguridad.

Las prácticas de escalada en general y los nudos en particular no sólo constituyen contenidos propios del Profesorado en Educación Física de la sede Bariloche de la Universidad Nacional del Comahue, sino que además se incorporan de manera explícita como núcleos temáticos en el área de Educación Física del Diseño Curricular de la educación secundaria de la provincia de Río Negro desde el año 2007 (Palacio, 2019). Así mismo en las recomendaciones para la elaboración de planes de estudios de profesorados de Educación Física del Ministerio de Educación de la Nación Argentina del año 2012, se incorpora una mirada amplia de la Educación Física que atiende a las características regionales culturalmente y territorialmente por lo que incorporan la práctica de la escalada como un posible eje a desarrollar. (Ministerio de Educación, 2012, p.18). Si a ello sumamos la incorporación de la escalada como deporte olímpico, podemos ver la importancia de los estudios que como el que acabamos de presentar relevan experiencias concretas de enseñanza, y evaluación en el contexto de la Educación Física.

En este sentido, desde el proyecto en general y este artículo en particular, se pretende aportar al marco referencial teórico relativo a las prácticas pedagógicas del Andinismo en la Educación Física en el escenario de la formación docente universitaria y en el ámbito profesional, procurando contribuir con el desarrollo de los conocimientos desde una indagación atenta a las particularidades del campo y a partir de la construcción de vínculos entre la teoría y la práctica. Este estudio, a su vez, puede constituirse en una fuente de datos e información para la construcción de propuestas alternativas y transformadoras para pensar, enseñar y mirar la cotidianeidad de las prácticas de escalada en marcos de seguridad y gestión de riesgo.

Se intenta ofrecer nuevas explicaciones de las prácticas evaluativas desplegadas en las prácticas pedagógicas de escalada para comprender los nuevos desafíos que se vislumbran en su enseñanza y lograr mejores intervenciones docentes en pro de la mejora de los aprendizajes. El diseño de dispositivos de evaluación vinculados a la investigación, permiten un registro sistemático que valida las decisiones y expresa cuantitativamente los datos que podrán retroalimentar las prácticas de formación docente.

\section{REFERENCIAS BIBLIOGRÁFICAS}

Achilli, E. (2002). Investigación y formación docente. III Encuentro Nacional de Docentes que hacen investigación educativa. Santa Fe: CTERA.

Ander Egg, E. (1993). Técnicas de investigación social. Buenos Aires: Magisterio del Río de la Plata.

Anijovich, R. (2010). La evaluación significativa. Buenos Aires: Paidós.

Anijovich, R. y Cappelletti, G. (2017). La evaluación como oportunidad. Buenos Aires: Paidós. 
Da Silva, T. (1995). Curriculum e identidad social. Territorios contestados. Buenos Aires: Departamento Ciencias de la Educación FFyL UBA.

Goicochea, M. y Lopez, E. (2016). Las prácticas de andinismo de Educación Física. Bariloche: Eduardo Hugo Lopez ediciones.

Hernández Sampieri, R, Fernández Collado, C. y Baptista Lucio, P. (2014). Metodología de la investigación. México: Mc Graw Hill.

Ministerio de Educación (2012). Proyecto demejora para la formación inicial deprofesores de nivel secundario: Educación Física. Argentina: Secretaría de Políticas Universitarias e Instituto Nacional de Formación Docente.

Núñez, T. (2005). Los mejores nudos de escalada. Madrid: Desnivel.

Palacio, M. (2019) Trekking y escalada en el Profesorado de la Universidad Nacional del Comahue. De la práctica a la enseñanza. Neuquén: EDUCO.

Raleigh, D. (1999). Nudos y cuerdas para escaladores. Madrid: Desnivel.

Ron, O. y Fernández, M. (2017). (En)Tramado. Educación Física y formación docente universitaria. Neuquén: Educo.

Steiman, J. (2008). Más didáctica (en la educación superior). Buenos Aires: Miño y Dávila.

Schubert, P., y Stockl, P. (2007). Montaña y Seguridad. Madrid: Desnivel.

\section{Notas}

1 Directora Marisa Fernández, Codirector Eduardo López

2 http://www.educacionyandinismo.com.ar

\section{BY-NC-SA}

